The book is certainly not easy to read, partly as a result of a rather clumsy prose style and the use of too wido a variety and size of type characters. There is a large number of useful diagrams, but some of these, for example $4.57(\mathrm{c}), 4.95$, and $8.18(\mathrm{c})$, are rather confused.

The most serious fault of the book is that it attempts to span too great a range of knowledgo and understanding. This raises difficulties which are particularly evident in the chapter on nuclei as quantum-mechanical systems. Here quantum numbers are introduced and explained in detail, including out of date notations, but reference is made to the Pauli exclusion principle, Fermi-Dirac and Bose-Einstein statistics without any explanation at all. The concept of isospin is dealt with very briefly although there is a fairly detailed discussion of isobaric analogue states later in the chapter, and the use of spin and isospin operators is not mentioned at all in this chapter. In all the theoretical sections, the underlying physical principles are rarely isolated and examined with clarity and insight.

DAPHNE F. JACKSON

\section{BASIC TEXT ON PLASMA PHYSICS}

\section{Nonlinear Plasma Theory}

By R. Z. Sagdeev and A. A. Galeev. Revised and edited by T. M. O'Neil and D. L. Book. (Frontiers in Physics.) Pp. xii + 122. (Benjamin: New York and Amsterdam, 1969.) n.p.

THIs book is based on a course of lectures given by the authors at the International Centre for Theoretical Physics at Trieste. The notes of this course, originally taken by the editors, have subsequently been revised and edited in collaboration with the authors.

The book gives an account of the theory of weakly non-linear plasmas (weak turbulence) and is conveniently divided into three sections. The first deals with the nonlinear interaction of waves. The authors start by discus. sing a simple example of three interacting waves and base the subsequent more general theory on this model. By introducing simple quantum concepts they succeed in giving a very simple interpretation of the effects even when applied to realistic situations. In this section of the book, a macroscopic approach to plasma physics is used, so the general methods developed are applicable to other fields of physics, particularly to those that are based on fluid type equations.

In the second section the resonant interaction of particles and waves is discussed, the so-called quasi-linear theory. Both three-dimensional effects and changes due to magnetic field fluctuations are discussed.

The final section is devoted to a discussion of the resonant interaction of particles with waves that themselves have been produced by the non-linear effects discussed in the first section. Thus the results of the first two sections are brought together to give the final non-linear theory. Mode-mode coupling effects and non-linear Landau damping are discussed.

Throughout the book the basic theory is well illustrated by examples which themselves are of importance in plasma physies. The book is addressed to plasma physicists and some familiarity with the linear theory of this subject is needed, in particular to understand fully the last two sections of this book. The first section gives an extremely good discussion of wave-wave interaction effects, however, which can be appreciated without this prior background and should be valuable to those working in other branches of physics.

The printing and layout of the book are in general very good, though the over-use of primed suffices, a notation which is prone to misprints, distract a little from what otherwise is an excellent book. I highly recommend it as a basic text on weakly non-linear plasma physics.

GeORge Rowlands

\section{ATOMIC COLLISIONS}

Electronic and lonic Impact Phenomena

Second edition in four volumes. By H. S. W. Massey, E. H. S. Burhop and H. B. Gilbody. Vol. 1: Electron Collisions with Atoms. Pp. $\mathrm{xx}+1-664+20$. Vol. 2: Electron Collisions with Molecules and Photoionization. Pp. xviii $+665-1294+21$. (Interuational Series of Monographs on Physics.) (Clarendon Press: Oxford; Oxford University Press: London, June 1969.) 200s each volume. WhEN the first edition of this well known monograph appeared in 1952, the authors managed to survey the whole field of atomic collision phenomena in a single, substantial volume of some six hundred pages. Such has been the pace of the development in the intervening years that the new edition will contain no less than four volumes cach comparable in size to the old one, and this has been achieved only at the expense of omitting certain topies entirely, notably the discussion of phenomena occurring at surfaces. The tremendous expansion that has occurred in the subject has been, in part, a response to the need in many branches of physics, notably in astrophysics, plasma physics and atmospheric physics, for accurate values of reaction rates for various atomic collision processes. The developments in experimental techniques, often springing from methods pioneered in the fields of nuclear and high energy physics, have made possible accurate absolute measurements of many differential and total cross-section for the first time. Perhaps the most interesting and fundamental advance in the theoretical interpretation of the data that has occurred since the first edition appeared has been the recognition of the important part that metastable complexes, formed from the colliding atoms or ions, play in many collision processes. The existence of these metastable states, observed as enhancements or resonances in the scattering cross-section, has been known from the $1930 \mathrm{~s}$, but at that time the profound effect that these levels have on excitation cross-sections in electron collisions with atoms or molecules and on many reaction rates for atom-ion collisions was not realized.

All these new phenomena, together with the many developments in the older parts of the subject, are reviewed in considerable detail, but with admirable clarity, in the new edition. The two published volumes cover the topics of electron collisions with atoms or ions (volume one) and electron collisions with molecules and photoionization (volume two). Collisions involving the scattering of ions or atoms by atoms or molecules will be treated in the third and fourth volumes. The authors belong to that very small group of physicists who can write with equal authority and experience on both theoretical and experimental topics, and in each volume they have provided a remarkably complete and balanced survey of the experimental techniques concerned and the results obtained, together with an illuminating discussion of the physical principles involved and the theoretical background. No attempt has been mado in the new volumes to list the experimental data completely, but all the really important results are described in full and most cross-sections of fundamental interest can be obtained from the book without reference elsowhere.

As this book is primarily an advanced treatise, it will naturally be of greatest value to active research workers in the field and to those working in related fields for which a knowledge of reaction rates is required, but at the samo time the book is so readable and treatment so clear that it can be recommended to oven the most junior research student as a source of reference.

The quality of the production is excellent and the mathematical text and the diagrams are very clear, something so often sadly lacking in modern scientific publishing. The index, which is of prime importance in works of reference of such length, is particularly good and it is easy to locate not only the material describing a particular experiment or technique, but also to find what is known about collision 